\title{
Über die (Un-)Kenntnis der ausländischen Pädagogik in Deutschland, dargestellt am Beispiel der pädagogischen Literatur Frankreichs, Italiens und Spaniens*
}

\author{
WALTRAUD HARTH-PETER
}

Anläßlich des 25. Jahrestages der Gründung von CESE erscheint es reizvoll, wieder einmal ${ }^{1}$ nach der tatsächlichen Kenntnis ausländischer Pädagogik in Deutschland zu fragen, weniger um die unermüdlichen Anstrengungen und Bemühungen des Geburtstagskindes zu überprüfen oder gar in Frage zu stellen, auch nicht um in naiver Absicht die reifen Früchte dieser zweifelsohne ertragreichen Arbeit der internationalen Gesellschaft zu ernten, sondern um durch eine neue, bibliometrisch erschlossene Bestandsaufnahme den Umfang des Wissens um das pädagogische Gedankengut unserer der romanischen Sprachkultur ${ }^{2}$ angehörenden Nachbarn festzustellen oder gar die Präsenz ihres Denkens in der deutschen Pädagogik nachzuweisen.

Im folgenden wird das Ergebnis der bibliometrischen Analyse ${ }^{3}$ zusammengefaßt und kurz kommentiert.

Das Augenmerk unserer Untersuchung liegt dabei auf der Rezeption der für ihr Land maßgeblichen Pädagogen und ihrer Ideen und nicht auf solchen Arbeiten, die es sich zur Aufgabe machen, Bildungssysteme in vergleichender Absicht zu beschreiben und dem deutschen Leser bekannt zu machen, auch nicht auf solchen wissenschaftlichen Anstrengungen, die den internationalen Vergleich in wissenschaftstheoretischer Absicht für eine erziehungswissenschaftliche Grundlagenreflexion gebrauchen wollen ${ }^{4}$.

Interessant erschien uns bei der Ermittlung der Daten auch die Frage, ob das durch unsere Analyse Gewonnene auf eine systematische Erschließung der ausländischen Pädagogik hinzuführen scheint, die uns dann erst ein umfassendes Bild des pädagogischen Gedankengutes unserer Nachbarländer zu liefern vermöchte, oder ob die Übersetzungen und Bearbeitungen einzelner Pädagogen oder pädagogischer Fragestellungen eher dem Zufall oder anderen unabwägbaren Faktoren zu verdanken sind.

Neben unserem besonderen Erkenntnisinteresse können wir es nicht unterlassen, darauf hinzuweisen, daß die Auseinandersetzung mit der Pädagogik der romanischen Länder vor allem im Bereich der institutionell-organisatorischen Erziehung mit ihren je speziellen Problemfeldern stattgefunden, und sich in den letzten 15 Jahren zusehends - zumindest für Frankreich - verdichtet hat.

\footnotetext{
* Kurzvortrag anläßlich des Sonderkongresses der Comparative Education Society in Europe (CESE) vom 3.-6. Oktober 1986 in Garda (Italien)
} 
Als Bernard Trouillet in seinem 1973 erschienenen Buch über "Die Sekundarschule in Frankreich. Ideologie, Probleme, Reformen"5 erstaunt die Frage an den Anfang stellte: "Sind Deutsche und Franzosen eigentlich Nachbarn?" 6 , war die Kenntnis des französischen Bildungswesens in Deutschland in der Tat eher spärlich und beschämend im Hinblick auf die deutsch-französische Verständigung, auch wenn während der 68er-Bewegung der Gedankenaustausch zumindest über die Mißstände der Universitäten, aber auch die Unbeweglichkeit der beiden nationalen Schulsysteme angesichts der politischen Forderungen der Bewegung unter denjenigen Kommilitonen intensiviert wurde, die der französischen Sprache mächtig und die verschiedenen Reformvorschläge und Überlegungen unterschiedlichster theoretischer Herkunft zur Kenntnis zu nehmen bereit waren. Als schöne Früchte dieser "grenzenlosen" bzw. grenzübergreifenden Kommunikation sind sicherlich die Dissertationen von Jürgen Schriewer über "Die französischen Universitäten 1945-1948. Probleme Diskussionen - Reformen. ${ }^{\text {7 }}$ und von Gisela Knaup über "Das Collège d'Enseignement Sécondaire (CES). Entstehung, Struktur, Entwicklungstendenzen eines französischen Sekundarschultyps. ${ }^{\prime 8}$ zu werten. Beide Autoren haben eine Entwicklung eingeleitet, die innerhalb der Vergleichenden Forschung den Bereich der institutionell-organisatorischen Erziehung mit (sozial-)wissenschaftlicher Akribie durchschaubar und für deutsche Erziehungswissenschaftler faßbar machte, und sie haben somit dazu beigetragen, der Vergleichenden Erziehungswissenschaft jene sozialwissenschaftlich orientierte thematische Priorität zu verleihen, die sie - wenn man internationale oder vergleichende Zeitschriften $^{9}$ aufmerksam liest - bis heute nicht verloren hat ${ }^{10}$.

Stellte man die Frage Trouillets auch im Hinblick auf die Rezeption ausländischer Pädagogen und ihres Gedankengutes, dann müßte man allerdings für die 15 Jahre, die seit Erscheinen seines Buches vergangen sind, ein sehr dunkles Bild zeichnen, da Kenntnisse über die Pädagogen der romanischen Länder und ihre Ideen nach unseren Untersuchungen im Grunde gar nicht und wenn, allenfalls höchst sporadisch und oft rein zufällig Eingang in das deutsche pädagogische Denken gefunden haben. Einen ersten Versuch, die Wechselbeziehung zwischen der deutschen und französischen Kultur und Sprache herzustellen, unternahm ebenfalls Trouillet in seinem 1981 erschienenen Buch mit dem Titel "Das deutsch-französische Verhältnis im Spiegel von Kultur und Sprache", das trotz seines Gewichts für unsere besondere Fragestellung nur am Rande von Bedeutung ist, da es sich vor allem mit der Vermittlung von Sprache und Kultur des Nachbarn im jeweils anderen Schulwesen beschäftigt: Trouillet stellt zunächst in einer kultur- und bildungshistorischen Analyse die deutsch-französischen Beziehungen seit dem Mittelalter dar; danach untersucht er die Stellung der Nachbarsprache im deutschen und französischen Schulwesen, wobei er "sowohl die fremdsprachenpolitischen als auch die schuladministrativen und -statistischen Aspekte in Betracht" zieht; und 
schließlich konzentriert er sich auf die "im schulischen Alltag zu sehr vernachlässigte Kulturkunde“, um anhand einer „Bestandsaufnahme einiger mit dem Fremdsprachenunterricht zusammenhängenden Probleme und Aufgaben" die Bedeutung der "kulturellen Dimensionen" und des "sozialkulturellen" Auftrages des Fremdsprachenunterrichts hervorzuheben (vgl. Trouillet 1981, S. XIV).

Interessant ist zunächst, daß die Erscheinungsorte der Schriften über die Pädagogik des Auslands ebenso begrenzt sind wie die Anzahl der Veröffentlichungen, so daß sich fast „Hochburgen “ für Internationale Pädagogik herauskristallisieren, zu denen - betrachtet man die Themen der Dissertationen und der Übersetzungen unseres Untersuchungszeitraumes - neben Frankfurt am Main, Tübingen, Köln, Münster und Hannover insbesondere die Universität Würzburg ${ }^{11}$ gehört. Im Vergleich zu Schriften, die sich mit Fragen des Bildungswesens und der Bildungspolitik der Nachbarländer beschäftigen, bleibt die Zahl derjenigen Arbeiten, die die Auseinandersetzung mit "ausländischen" Pädagogen und deren Ideen angestrebt haben, verschwindend gering. Auch die Zahl der Übersetzungen ist erschreckend niedrig ${ }^{12}$.

Für Spanien etwa kann die klägliche Bilanz gezogen werden, daß, abgesehen von den gesammelten Werken des Kulturphilosophen José Ortega y Gasset (1883-1955), überhaupt kein Gesamtwerk eines spanischen Pädagogen in deutscher Sprache vorliegt. Es steht zu vermuten, daß Ortega y Gassets Schriften vor allem deshalb übersetzt wurden, weil einmal der Einfluß Diltheys und Nietzsches auf das Denken Ortega y Gassets, zum anderen dessen leidenschaftlich vertretene Idee eines neuen Europa und zum dritten seine Studien in Deutschland ein Ignorieren seiner Gedanken in unserem Land unmöglich gemacht haben, gehört er doch ohnehin fast zu den "deutschen Denkern". Außer einer 1969 erschienenen Studie über "Das öffentliche Elementarschulwesen in Spanien "13 von Nils Hole, die nach eigener Angabe auf wenig erschlossenes Material zur systematischen Erfassung des spanischen Erziehungssystems zurückgreifen konnte, ist 1982 ein von Maria Malescu verfaßtes Buch über die „Bildungsreform in Spanien. 1970-1980"14 und 1983 ein kurzer Aufsatz von Goldine B. Witte über "Spaniens Weg zu einem modernen Erziehungssystem"15 erschienen. Während Hole auf einige bekannte spanische Pädagogen „liberaler" (Francisco Giner de los Ríos, Manuel B. Cossío, Joaquín Costa) und "traditionalistischer Erziehungsbewegung" (Marcelino Menédez y Pelayo, Rufino Blanco y Sánchez, Ramón Ruiz Amado de Contreras S.J., Andrés Manjón) hinweist, liefert der Aufsatz von Witte eine Darstellung der Entwicklung des spanischen Bildungswesens ab 1951 bis heute. 1985 ergänzte Maria Malescu ihre Studien über die spanischen Erziehungsinstitutionen durch ein Werk über Geschichte und Gegenwart der spanischen Universität ${ }^{16}$.

Nahezu alleinstehend erscheint daher für die Rezeption spanischer Pädagogik die 1986 in Würzburg abgeschlossene Dissertation von Margarita Schweizer 
über "Andrés Manjón. Ein spanischer und christlicher Reformpädagoge“17, die es nicht nur verstanden hat, das Wirken und Denken dieses Pädagogen in die pädagogische Diskussion seiner Zeit einzuordnen, sondern darüber hinaus den Deutschen einen ersten Einblick in die spanische Pädagogik der Jahrhundertwende zu geben. Durch diese Arbeit wie auch durch den regen Austausch zwischen der Universität Würzburg und ihrer Partneruniversität Salamanca scheint hier die von allen drei romanischen Sprachen größte sprachliche Barriere abgebaut zu werden; ein Zugang zur spanischen Pädagogik öffnet sich erst ganz allmählich; nicht zuletzt durch Veröffentlichungen pädagogischer Gedanken des der Universität Würzburg durch einen Forschungsauftrag verbundenen Salmantiner Professors José Ortega Esteban in deutscher und Winfried Böhms in spanischer Sprache ${ }^{18}$.

Prüft man die Kenntnis der italienischen Pädagogik in Deutschland, so könnte man - horribile dictu - fast den Eindruck gewinnen, als seien Maria Montessori und Don Bosco allein repräsentativ für Theorie und Praxis italienischer Pädagogik gewesen -, oder es könnte scheinen, als ob die radikale Provokation Montessoris für eine Pädagogik vom Kinde aus oder die caritativsozialpädagogische Arbeit Don Boscos international mehr Aufsehen erregt haben als die eher bescheiden zu nennende theoretische Fundierung ihrer Erziehungsgedanken. Auffallend dabei ist, daß sie in Italien selbst nur wenig bekannt und jedenfalls nicht als nationale Erzieher anerkannt sind ${ }^{19}$. Neben diesen (vermeintlichen) "Galionsfiguren" italienischer Pädagogik fallen zahlenmäßig Übersetzungen des Werkes von Benedetto Croce $^{20}$ und Auseinandersetzungen mit ihm auf; dabei ist Croce freilich ein Autor, der mehr aufgrund seines philosophischen Gedankengutes und seiner antiklerikalen Kulturkritik denn als Pädagoge rezipiert wurde. Aufnahme in die deutsche Literatur fanden einzelne Schriften von Catalfamo ${ }^{21}$, Flores d'Arcais ${ }^{22}$, Gentile ${ }^{23}$, Gram$\mathrm{sci}^{14}$, Laporta ${ }^{25}$, Lombardo Radice ${ }^{26}$, Volpicelli ${ }^{27}$, die allerdings in ganz unterschiedlichem Umfang in die deutsche Sprache übertragen wurden. Das Verdienst, den bedeutenden und von der deutschen Pädagogik sträflich vernachlässigten Giambattista Vico vorgestellt zu haben und für die deutsche Pädagogik fruchtbar zu machen, kommt Clemens Menze ${ }^{28}$ und Winfried Böhm ${ }^{29} \mathrm{zu}$.

Die erste Textsammlung von bedeutenden italienischen Pädagogen stellt „Die italienische Pädagogik des 20. Jahrhunderts" (1979), herausgegeben von Giuseppe Flores d'Arcais und Winfried Böhm, dar; sie kann als bisher einzige umfassende Orientierung über die italienische Pädagogik unseres Jahrhunderts gelten.

Die Kenntnis französischer Pädagogik ist bei weitem breiter als die der beiden anderen Länder. Aber hier handelt es sich vor allem um die Darstellung und um den Vergleich der Bildungssysteme und weniger um einen vertieften Einblick in das Erziehungsdenken unseres Nachbarlandes. Auch der Versuch, den interkulturellen Vergleich zwischen französischer Erziehungswissenschaft 
und deutscher Pädagogik auf der wissenschaftstheoretischen und methodischen Ebene zu führen, wurde eher im Sinne einer "Aufarbeitung divergierender innerdeutscher Wissenschaftsentwicklungen ${ }^{\prime 30}$ und einer Befreiung der durch die geisteswissenschaftliche Tradition in ihrem Methodenverständnis vermeintlich (!) eingeengten deutschen Pädagogik verstanden ${ }^{31}$, denn als ein Sich-Einlassen auf das Denken anderer Nationen, um durch eine solche Konfrontation eine Integration ausländischer Gedanken in unser pädagogisches Denken einzuleiten, ohne dieses von vornherein durch methodologische Flügelkämpfe einer positivistischen Erziehungswissenschaft auszuliefern.

Die meisten Schriften von und über französische Pädagogen findet man verständlicherweise $z u$ Rousseau ${ }^{32}$, den man jedoch als internationalen und nicht nur als französischen Pädagogen betrachten muß. Mehrere Artikel und Monographien beschäftigen sich mit Célestin Freinet ${ }^{33}$ und dessen eigenwilliger Schulpraxis (einen "Boom" erlebt er z. Z. unter sonderpädagogischer Rücksicht); mit Abstand folgen Arbeiten über Fénelon ${ }^{34}$, Condorcet ${ }^{35}$, Montaigne ${ }^{36}$, Kergomard ${ }^{37}$, Alain ${ }^{38}$ und Ariès ${ }^{39}$. Einige Dissertationen der letzten Jahre trugen dazu bei, daß einzelne französische Erziehungsgedanken bekannt wurden: 1974 beschäftigte sich Frauke Stübig mit der éducation commune der Französischen Revolution ${ }^{40} ; 1976$ setzte sich Berthold Langer ${ }^{41} u$. a. mit dem Bildungsbegriff Condorcets auseinander. 1983, 1984 und 1986 beschäftigten sich drei Dissertationen, alle vorgelegt an der Universität Würzburg, mit französischer Pädagogik: Gabriele Weigand ${ }^{42}$ stellte die pédagogie institutionnelle vor; Hans-Peter Merz-Chrétien ${ }^{43}$ untersuchte die Pädagogik Eugène Dévauds und Waltraud Harth ${ }^{44}$ die Anfänge der Neuen Erziehung in Frankreich. Ergänzt werden die Würzburger Studien zur internationalen Pädagogik von der von Giuseppe Flores d'Arcais und Winfried Böhm ${ }^{45}$ herausgegebenen Anthologie über „Die Pädagogik der frankophonen Länder im 20. Jahrhundert".

Zusammenfassend kann man sagen, daß trotz großer Bemühungen seitens der Vergleichenden Erziehungswissenschaft und trotz des Wirkens internationaler Vereinigungen eine Rezeption romanischer Pädagogik in Deutschland nur in vereinzelten und fast sporadisch zu nennenden Fällen festzustellen ist. Die Gründe dafür mögen in den immer noch vorhandenen Sprachbarrieren liegen, in der eigenen, im Vergleich zu den Nachbarländern stark divergierenden Entwicklung der deutschen Pädagogik (seit dem Dritten Reich) und nicht zuletzt in dem vorwiegenden Erkenntnisinteresse der sozialwissenschaftlich orientierten deutschen Erziehungswissenschaft an institutionell-organisatorischer Erziehung und an wissenschaftstheoretischen Fragestellungen. 


\section{Anmerkungen}

1 Vgl. dazu:

Die Präsenz der italienischen Kultur im deutschen und die der deutschen Kultur im italienischen Sprachraum seit 1945. Akten der V. internationalen Tagung deutschitalienischer Studien vom 1. bis 8. April 1964 in Meran. Hrsg. von dem DeutschItalienischen Kulturinstitut in Südtirol. Meran 1968.

Winfried Böhm: Einleitung zu Winfried Böhm/Giuseppe Flores d'Arcais: Die italienische Pädagogik des 20. Jahrhunderts. Stuttgart 1979, S. 8-35.

Winfried Böhm: Vorwort zu Winfried Böhm/Giuseppe Flores d'Arcais: Die Pädagogik der frankophonen Länder im 20. Jahrhundert. Stuttgart 1980, S. 810.

Kurt Gerhard Fischer: Aspekte der italienischen Pädagogik der Gegenwart. In: Zeitschrift für Pädagogik 1966, S. 482-502.

2 Wir wählen hier die romanischen Länder exemplarisch aus, weil ihnen von der historischen Bedeutung für die europäische Kultur - auch die europäische pädagogische Kultur - und von der innovatorischen Kraft der pädagogischen Entwürfe dieses Sprachraums her gewiß ein größeres Gewicht zukommt als etwa dem nordischangelsächsischen Sprachraum.

3 Als Grundlage für die Datenerhebung dienten uns je 25 Jahrgänge der bekanntesten pädagogischen Zeitschriften, die nach ihren Beiträgen über das pädagogische Gedankengut der drei Länder befragt wurden. Weiter durchforsteten wir ausgewählte Werke zur Geschichte der Pädagogik nach Vertretern der romanischen Pädagogik und fragten nach den an deutschen Universitäten abgeschlossenen Dissertationen, die sich mit der Pädagogik dieser Länder befaßten. Schließlich suchten wir nach Monographien von französischen, italienischen und spanischen Pädagogen, die in den letzten 25 Jahren ins Deutsche übersetzt wurden.

An Zeitschriften wurden verwendet:

Bildung und Erziehung.

Die Deutsche Schule.

Internationale Zeitschrift für Erziehungswissenschaft. International Review of Education. Revue international de pédagogie.

Neue Sammlung.

Pädagogische Rundschau.

Vierteljahrsschrift für wissenschaftliche Pädagogik.

Zeitschrift für Pädagogik.

Werke zur Geschichte der Pädagogik:

Th. Ballauff/KI. Schaller: Pädagogik. Eine Geschichte der Bildung und Erziehung. 3 Bände. Freiburg/München 1969, 1970 und 1973.

Herwig Blankertz: Die Geschichte der Pädagogik von der Aufklärung bis zur Gegenwart. Wetzlar 1982.

Fritz Blättner: Geschichte der Pädagogik. Heidelberg ${ }^{13} 1978$.

U. v. der Burg/R. Hülshoff: Geschichte der Pädagogik. Stationen von der Aufklärung bis zur Gegenwart. Düsseldorf 1979.

Theo Dietrich: Geschichte der Pädagogik in Beispielen. 18.-20. Jahrhundert. Bad Heilbrunn 1970.

J. v. Driesch/J. Esterhues: Geschichte der Erziehung und Bildung. 2 Bände. Paderborn ${ }^{5} 1960-61$.

Hans Michael Elzer: Bildungsgeschichte als Kulturgeschichte. Ratingen 1965.

Ulrich Hermann (Hrsg.): Historische Pädagogik. Weinheim/Basel 1977.

Karl Knoop/Martin Schwab: Einführung in die Geschichte der Pädagogik. Heidelberg 1981. 
Werner Raith: Von Comenius bis ins 20. Jahrhundert. Geschichte der neuzeitlichen Pädagogik. Starnberg 1968.

Albert Reble: Geschichte der Pädagogik ${ }^{13} 1981$ (TB-Ausgabe nach 12. Aufl.v. 1975) Josef Speck (Hrsg.): Geschichte der Pädagogik des 20. Jahrhunderts. 2 Bände. Stuttgart 1978.

4 Vgl. dazu z. B. Jürgen Schriewer: „Erziehung und Kultur”. Zur Theorie und Methodik Vergleichender Erziehungswissenschaft. In: Brinkmann/Renner: Die Pädagogik und ihre Bereiche. Paderborn 1982, S. $185 \mathrm{ff}$.

5 Bernard Trouillet: Die Sekundarschule in Frankreich. Ideologie, Probleme, Reformen. Weinheim 1973.

6 Vgl. dazu auch W. Hörner: Bildungsreform und pädagogische Wissenschaft in Frankreich. In: Bildung und Erziehung 28 (1975), S. 387-399

7 Jürgen Schriewer: Die französischen Universitäten 1945- 1948. Probleme - Diskussionen - Reformen. Würzburger Arbeiten zur Erziehungswissenschaft, Bad Heilbrunn 1972.

8 Gisela Knaup: Das Collège d'Enseignement Sécondaire (CES). Entstehung, Struktur, Entwicklungstendenzen eines französischen Sekundarschultyps. Hannover 1974.

9 Comparative Education.

Comparative Education Review.

Education comparée.

European Journal of Education. (Bis 1979: Paedagogica Europaea).

Internationale Zeitschrift für Erziehungswissenschaft. International Review of Education. Revue internationale de pédagogie.

Rassegna di Pedagogia. Pädagogische Umschau.

10 Das Erkenntnisinteresse Jürgen Schriewers liegt heute jedoch mehr auf der Vergleichenden Erziehungswissenschaft als einem Vehikel wissenschaftstheoretischer Erwägungen. Vgl. Jürgen Schriewer: Pädagogik - ein deutsches Syndrom? In: Zeitschrift für Pädagogik 1983, S. 359-389 und ders. a. a. O.

11 Allein sechs von insgesamt 24 Dissertationen, die sich mit der Pädagogik romanischer Länder befassen, kann das Institut für Pädagogik der Universität Würzburg für sich beanspruchen. (Neben den Dissertationen existieren einige Diplomarbeiten, die sich mit italienischen und französischen Pädagogen beschäftigten.) Anzumerken bleibt noch, daß ein Viertel aller hier einschlägigen Dissertationen im Bundesgebiet Rousseau oder Montessori zum Thema hat.

Darüber hinaus kann die seit 1982 in Würzburg erscheinende Reihe "Internationale Pädagogik", herausgegeben von Winfried Böhm, bereits 14 Schriften von oder über ausländische Pädagogen verzeichnen.

12 Bevorzugt werden die Klassiker einzelner Länder übersetzt. Auffallend dabei ist, daß die Nachbarwissenschaften der Pädagogik wie Philosophie, Psychologie und Soziologie wesentlich rühriger in der Verbreitung ausländischen Gedankenguts sind.

13 Nils Hole: Das öffentliche Elementarschulwesen Spaniens. Ratingen 1969.

14 Maria Malescu: Bildungsreform in Spanien. 1970-1980. Weinheim 1982.

15 Goldine B. Witte: Spaniens Weg zu einem modernen Erziehungssystem. In: Die Deutsche Schule 1983, S. 63-73.

16 Maria Malescu: Die spanische Universität in Geschichte und Gegenwart. Köln 1985.

17 Margarita Schweizer: Andrés Manjón. Ein spanischer und christlicher Reformpädagoge. (Diss.) Würzburg 1986; erscheint als Buch unter dem gleichen Titel im Verlag Königshausen \& Neumann, Würzburg 1987. 
18 José Ortega Esteban: Ein Fall wissenschaftlicher Inkulturation. Der Einfluß der europäischen Pädagogik in Spanien. In: Rassegna di Pedagogia. 42, 1984, S. 225-236. In Vorbereitung befindet sich: José Ortega Esteban und Winfried Böhm: Die spanische Pädagogik im 20. Jahrhundert (erscheint voraussichtlich $1988 \mathrm{im}$ Verlag Königshausen \& Neumann, Würzburg) sowie Winfried Böhm, José Ortega Esteban, Miguel Petty: Die hispano-amerikanische Pädagogik des 20. Jahrhunderts.

Winfried Böhm: Antropología y educación. Córdoba (Argentinien) 1983.

Ders.: La reforma de la educación preescolar como problema internacional. In: Enfoques Educacionales 5 (1979), S. 30-39.

Ders.: La tarea polifacética del maestro. In: Perfiles Educativos 12 (1981), S. 22-29.

Ders.: La educación de la persona. Buenos Aires 1982.

Ders.: El declino de la universidad. Salamanca 1985.

$19 \mathrm{Vgl}$. Roberto Mazzetti: Giuseppe Lombardo-Radice tra l'idealismo pedagogico e Maria Montessori. Bologna 1958.

Die Arbeiten über Don Bosco in Deutschland sind übrigens weitaus zahlreicher als in Italien; ein Ähnliches wäre im umgekehrten Falle zu F. W. Foerster zu sagen.

20 Von Croce wurden in deutscher Sprache die Gesammelten Werke in 7 Bänden (1927-1930) veröffentlicht, außerdem einzelne Schriften.

21 Giuseppe Catalfamo: Ideologie und Erziehung. Aus dem Italienischen übersetzt von Liselotte Reich-Coregliano und Winfried Böhm. In der Reihe: Internationale Pädagogik. Hrsg. von Winfried Böhm. Band 9. Würzburg 1984.

22 Giuseppe Flores d'Arcais: Geschichte der Pädagogik und pädagogische Theorie. In: W. Böhm/J. Schriewer (Hrsg.): Geschichte der Pädagogik und systematische Erziehungswissenschaft. Stuttgart 1975, S. 11-20.

Ders./W. Böhm (Hrsg.): Die italienische Pädagogik des 20. Jahrhunderts. Stuttgart 1979.

Ders./W. Böhm (Hrsg.): Die Pädagogik der frankophonen Länder im 20. Jahrhundert. Stuttgart 1980.

${ }^{23}$ Die Übersetzungen von Gentiles Schriften haben in deutscher Sprache immerhin bereits eine ansehnliche Zahl erreicht.

24 Antonio Gramsci: Philosophie der Praxis. Frankfurt am Main 1967.

Ders.: Marxismus und Kultur. Frankfurt/Main.

Zu Gramsci:

Gerhard Roth: Gramscis Philosophie der Praxis. Düsseldorf 1972.

Ursula Schnieder: Versuch einer Darstellung der pädagogischen Prinzipien Antonio Gramscis. (Unveröffentlichte pädagogische Diplomarbeit. Universität Würzburg 1980).

25 Raffaele Laporta: Meine Pädagogik. In: Rassegna di Pedagogia 36 (1978), S. 5-29.

26 Zu Giuseppe Lombardo-Radice:

Sergius Hessen: Die scuola serena von G. Lombardo-Radice. In: Die Erziehung 6 (1931), S. 533-534.

27 Luigi Volpicelli: Pädagogik und Gesamtkultur. In: Erziehung zur Menschlichkeit. Festschrift für E. Spranger. Tübingen 1957. Unerwähnt bleiben kann hier Volpicellis Buch über die Sowjetische Schule, das auf deutsch vorliegt.

${ }^{28}$ Clemens Menze: Metaphysik, Geschichte, Bildung bei Giambattista Vico. Ein Kapitel aus der Geschichte der Pädagogik der Urteilskraft. In: Vierteljahrsschrift für wissenschaftliche Pädagogik 1985, S. 16-63.

29 Winfried Böhm: Theorie und Praxis. Eine Erörterung des pädagogischen Grundproblems. Würzburg 1985. 
$30 \mathrm{Vgl}$. Jürgen Schriewer, a. a. O. 1983, S. 359.

31 Auch Debesse spricht davon, daß Frankreich eigentlich keine reinen Pädagogen, sondern allein "Erziehungswissenschaftler" habe, die in den verschiedenen Erziehungswissenschaften zuhause sind.

32 Die Literatur zu Rousseau kann als bekannt vorausgesetzt werden.

33 Die Literatur zu Freinet kann der Arbeit von Gabriele Weigand: Erziehung trotz Institutionen? Die pédagogie institutionelle in Frankreich. In der Reihe: Internationale Pädagogik. Hrsg. von Winfried Böhm. Band 7. Würzburg 1983 entnommen werden. Zur Zeit entsteht unter der Leitung von Hans Jörg eine Arbeit über Freinet mit dem Arbeitstitel "Die philosophischen und sozialen Grundlagen der pädagogischen Ideen des Célestin Freinet".

34 Zu Fénelon:

Robert Spaemann: Reflexion und Spontaneität, Studien über Fénelon. Stuttgart 1963.

Martin Rang: Erziehung als Läuterung. Zu Spaemanns Fénelonstudie. In: Zeitschrift für Pädagogik 1965, S. 581-589.

35 Berthold Langer: Die Entwicklung eines gesellschaftstheoretisch fundierten Bildungsbegriffs bei Condorcet, Hege! und Marx. Diss. Universität München 1976. Heinz-Hermann Schepp: Freiheit und Gleichheit in Condorcets Plan einer Neuorganisation des Unterrichtswesens (1972). In: Zeitschrift für Pädagogik 1966, S. 144-152.

Ders./B. Michael (Hrsg.): Politik und Schule von der Französischen Revolution bis zur Gegenwart. Band 1. 1973.

Eine Habilitation über die erwachsenenpädagogische Bedeutung und die Wirkungen der bildungspolitischen Schriften von Condorcet wird zur Zeit von Detlef Oppermann (Saarbrücken) angestrebt.

36 Michel de Montaigne: Essays über Erziehung. Bad Heilbrunn 1964.

Ders.: Vom Schaukeln der Dinge. Berlin 1984.

Ders.: Zum Zeitvertreib und um die Phantasie zu tummeln. Zürich 1985.

Zu Montaigne:

Carlo Jenzer: Lebensnähe, Lebensferne und Realismus in den pädagogischen Ansichten von Michel de Montaigne. Bern 1969.

37 Waltraud Harth: Pauline Kergomard und die Entwicklung der Vorschulerziehung in Frankreich. In der Reihe: Internationale Pädagogik. Hrsg. von Winfried Böhm. Band 6. Würzburg 1983.

38 Emile-Auguste Chartier (Alain): Mars oder die Psychologie des Krieges. Frankfurt/Main 1983.

Ders.: Über die Erziehung, Paderborn 1964.

Ders.: Die Pflicht, glücklich zu sein. Frankfurt/Main 1975.

Ders.: Spielregeln der Kunst. Frankfurt/Main 1985.

Zu Alain:

Ludwig Schmidts: Alain. Bemerkungen zur Erziehung. In: Pädagogische Rundschau 1962, S. 91-98.

39 Philippe Ariès: Geschichte der Kindheit, München 1975.

Zu Ariès:

W. Hornstein/A. Flitner: Neue Literatur zur Geschichte des Kindes- und Jugendalters. U. a.: Philippe Ariès, L'enfant et la vie familiale sous l'ancien régime. In: Zeitschrift für Pädagogik 1965, S. 66-85. 
Klaus Arnold: Kind und Gesellschaft in Mittelalter und Renaissance. Beiträge und Texte zur Geschichte der Kindheit. In: Reihe Zebra. Schriften zur Kleinkind- und Vorschulerziehung, hrsg. von Winfried Böhm, Paderborn 1980.

40 Frauke Stübig: Erziehung zur Gleichheit. Die Konzepte der éducation commune in der Französischen Revolution. Diss. Ravensburg 1974.

41 Berthold Langer, a. a. O.

42 Gabriele Weigand, a. a. O.

43 Hans-Peter Merz-Chrétien: Ursprung und Leitmotiv der Pädagogik Eugène Dévauds. Diss. Würzburg 1984.

44 Waltraud Harth: Die Anfänge der Neuen Erziehung in Frankreich. In der Reihe: Internationale Pädagogik. Hrsg. von Winfried Böhm. Band 13. Würzburg 1986.

45 Winfried Böhm/Giuseppe Flores d'Arcais, a. a. O. 